\title{
Identificación de especies de micobacterias mediante espectrometría de masas (MALDI-TOF)
}

\section{Identification of mycobacteria species through mass spectrometry (MALDI-TOF)}

\author{
Samuel Contreras', David Rodríguez², Francisco Vera², María Elvira Balcells', Luis Celis', \\ Paulette Legarraga², Juan Carlos Román y Patricia García ${ }^{2}$
}

'Laboratorio de Microbiología, Servicio de Laboratorios Clínicos Red de Salud UC-CHRISTUS. Santiago, Chile.
Departamento de Laboratorios Clínicos, Escuela de Medicina, Pontificia Universidad Católica de Chile. Santiago, Chile.
${ }^{3}$ Departamento de Enfermedades Infecciosas del Adulto, Escuela de Medicina, Pontificia Universidad Católica de Chile. Santiago, Chile.

Financiamiento: Fondos del Departamento de Laboratorios Clínicos de la Escuela de Medicina de la Pontificia Universidad Católica de Chile. Conflicto de intereses: Ninguno

Recibido: 3 de mayo de 2019 / Aceptado: 19 de noviembre de 2019

\section{Resumen}

Introducción: Las enfermedades producidas por micobacterias son de gran importancia clínica y epidemiológica presentando el complejo Mycobacterium tuberculosis (MTBc) una morbi-mortalidad mayor que la producida por micobacterias no tuberculosas (MNTB). La identificación tradicional está basada en sus características fenotípicas mediante procesos laboriosos e incapaces en algunos casos de distinguir entre especies. Actualmente, la mayoría de las técnicas utilizadas se basan en métodos moleculares que tienen alta veracidad, pero son complejas y de alto costo. La espectrometría de masas con desorción/ionización láser asistida por una matriz asociada a tiempo de vuelo (MALDI-TOF MS) se basa en la comparación del espectro proteico producido con respecto al de una base de datos de referencia. Objetivo: Evaluar el rendimiento de MALDI-TOF MS en la identificación de micobacterias comparado con métodos moleculares: Material y Métodos: Se analizaron 28 aislados de nueve especies distintas mediante MALDI-TOF MS. Resultados: Se identificó correctamente 78,5\% de las aislados (22/28), concordante en $100 \%$ (9/9) de MNTB de crecimiento rápido, $60 \%(9 / 15)$ en las MNTB de crecimiento lento y $100 \%$ (4/4) de MTBc. Todas las especies no identificadas (6/6) pertenecen al complejo $M$. avium/intracellulare. Conclusión: MALDI-TOD MS es una metodología rápida, fácil y de bajo costo, con adecuada veracidad respecto a los métodos moleculares.

Palabras clave: micobacterias; espectrometría de masas; identificación bacteriana; MALDI-TOF MS.

\begin{abstract}
Background: Mycobacterial diseases are very important both clinically and epidemiologically. Mycobacterium tuberculosis complex (MTBc) infections confer higher morbidity and mortality rate than non-tuberculous mycobacteria (NTM) infections. Traditional species identification techniques are based on phenotypic characteristics which take a long time by laborious processes and in occasions are no conclusive. Currently, most used techniques are based on molecular methods, which are accurate but are expensive and complex. Matrix Assisted Laser Desorption/Ionization Time-of-Flight mass spectrometry (MALDI-TOF MS) is a simple, cheap and fast identification method based on comparing protein spectra with a reference database. Aim: To assess the performance of MALDI-TOF MS in the identification of MTBc and NTM, compared with molecular methods. Methods: For that purpose, 28 isolates of 9 different species were analyzed through MALDI-TOF MS. Results: $78.5 \%$ (22/28) of isolates were correctly identified, $100 \%(9 / 9)$ of rapidly growers NTM, $60 \%(9 / 15)$ of slow growing NTM and $100 \%(4 / 4)$ of MTBc. Every unidentified isolate (6/6) corresponded to M. avium/intracellulare complex. Conclusion: MALDI-TOF MS is fast, simple and cheaper than molecular methods and also has adequate accuracy.

Keywords: Mycobacteria; mass spectrometry; bacterial identification; MALDI-TOF MS.
\end{abstract}

\section{Correspondencia a:}

Patricia García Cañete

pgarciacan@uc.c 
sondas en tira por el Instituto de Salud Pública de Chile (19 aislados) y por el Colegio de Patólogos Americanos (CAP) (9 aislados).

as micobacterias son un grupo de microorganismos que causan un amplio espectro de enfermedades, tanto pulmonares como extra-pulmonares ${ }^{1,2}$. El complejo Mycobacterium tuberculosis (MTBc) corresponde a patógenos con una mayor morbi-mortalidad ${ }^{3}$. Las micobacterias no tuberculosas (MNT) ambientales, generalmente actúan como oportunistas en pacientes inmunocomprometidos, provocando gran variedad de entidades clínicas como afecciones cutáneas, ganglionares y pulmonares cuya incidencia global ha incrementado ${ }^{4,5}$. Sin embargo, existen MNT ambientales no relacionadas con enfermedades en humanos y que frecuentemente son contaminantes de muestra clínicas, por lo que es imperativo un método diagnóstico en la identificación de especies para conferir una significancia clínica a un aislado micobacteriano y, por lo tanto, otorgar un tratamiento y pronóstico adecuados.

La identificación tradicional de las micobacterias está basada en sus características bioquímicas y fenotípicas mediante procesos lentos, laboriosos, e incapaces en algunos casos, de distinguir entre especies. Actualmente, la mayoría de las técnicas usadas en la identificación de micobacterias están basadas en métodos moleculares (considerados como el estándar de oro) que tienen una gran veracidad, pero son de alto costo y generalmente están confinados a laboratorios de referencia.

La espectrometría de masas basada en Matrix-assisted laser desorption/ionization time-of-flight (MALDI-TOF) es una espectrometría de ionización suave que se basa en la comparación del espectro proteico producido por una muestra en particular, respecto al de una base de datos de referencia ${ }^{6}$ (Figura 1). MALDI-TOF ha sido utilizado en la identificación de bacterias y levaduras y se ha caracterizado por ser un método barato, rápido y simple que puede ser además ser implementado en laboratorios de microbiología general ${ }^{7-11}$.

\section{Objetivo}

Evaluar el rendimiento de MALDI-TOF en la identificación de MTB y MNTB comparado con métodos moleculares.

\section{Materiales y Métodos}

\section{Aislados estudiados}

Se analizaron 28 aislados de 9 especies distintas (24 MNTB y $4 \mathrm{MTBc}$ ), complejo M. tuberculosis (n: 4), Complejo M. avium/intracellulare (n: 11), complejo M. abscessus/chelonae (n: 4), M. fortuitum (n: 2), M. gordonae (n: 2), M. mucogenicum (n: 2), M. neoaurum (n: 1), M. kansasii (n: 1) y M. terrae (n: 1). La identificación fue confirmada mediante hibridación reversa con

\section{Extracción de proteínas}

Los aislados fueron obtenidos a partir de muestras clínicas o procedentes de control de calidad externo por parte del CAP y cultivadas en medio líquido (Mycobacteria Growth Indicator Tube MGIT, Becton Dickinson and Company, Sparks, MD, USA) o sólido (medio Lowenstein- Jensen, Becton Dickinson and Company, Sparks, MD, USA) a $37^{\circ} \mathrm{C}$. Las muestras fueron procesadas hasta su inactivación, en un laboratorio de nivel de bioseguridad 2, que cuenta con un gabinete de bioseguridad clase 2B (con salida al exterior), con todo el equipamiento al interior del laboratorio, con exclusa de acceso. En el caso de los cultivos líquidos, con tiempo máximo de 48 horas después de que el equipo automatizado (BD Bactec MGIT, Becton Dickinson and Company, Sparks, MD, USA) los clasificara como positivos, fue extraído $1,5 \mathrm{ml}$ desde el fondo del tubo, volumen que se centrifugó a $14.000 \mathrm{x} \mathrm{g}$ por $2 \mathrm{~min}$, reservándose el pellet resultante. Por otra parte, en los cultivos sólidos se trabajó directamente con una asada de $1 \mu \mathrm{L}$ de colonia. La masa bacteriana obtenida tanto de cultivos líquidos como sólidos fue suspendida en $300 \mu \mathrm{l}$ de agua grado cromatografía (Merck, Darmstadt, Germany) en un tubo de microcentrífuga de $1,5 \mathrm{ml}$ y calentada a $100^{\circ} \mathrm{C}$ durante $30 \mathrm{~min}$ en termo-bloque con el fin de inactivar a los microorganismos ${ }^{12}$. Posteriormente, se agregó $900 \mu \mathrm{l}$ de etanol grado cromatografía al 100\% (v/v) y se adicionaron entre 20 a $40 \mathrm{mg}$ de esferas de zirconia/sílica 0,5 mm (BioSpec Products Inc., OK, USA). Los tubos fueron agitados por 1 min en agitador mecánico, traspasándose el contenido por decantación a otro tubo de microcentrífuga evitando transferir las esferas. Inmediatamente, los tubos fueron centrifugados a $14.000 \mathrm{x} \mathrm{g}$ por 2 min y se eliminó el sobrenadante. Se centrifugaron nuevamente para eliminar todo el resto del líquido y se dejaron secar a temperatura ambiente al interior del gabinete de bioseguridad por $15 \mathrm{~min}$. Posteriormente, el proceso de extracción de proteínas se realizó con reactivos preparados en el laboratorio. Dependiendo del tamaño, el pellet fue disuelto entre 10 y $30 \mu \mathrm{l}$ de ácido fórmico al 70\% (v/v) (Merck, Darmstadt, Germany) mediante un agitador mecánico por 20 segundos. Finalmente, se agregó el mismo volumen de acetonitrilo al 100\% (v/v) (Merck, Darmstadt, Germany) homogenizándose mediante una corta agitación.

\section{Adquisición y procesamiento del espectro MALDI-TOF}

Los extractos proteicos fueron centrifugados a 14.000 $\mathrm{x}$ g por 2 min y el sobrenadante resultante fue cargado $1 \mu 1$ por duplicado sobre una placa de incidencia del láser 
Figura 1. Resumen del proceso analítico para la identificación de micobacterias por MALDI-TOF MS.

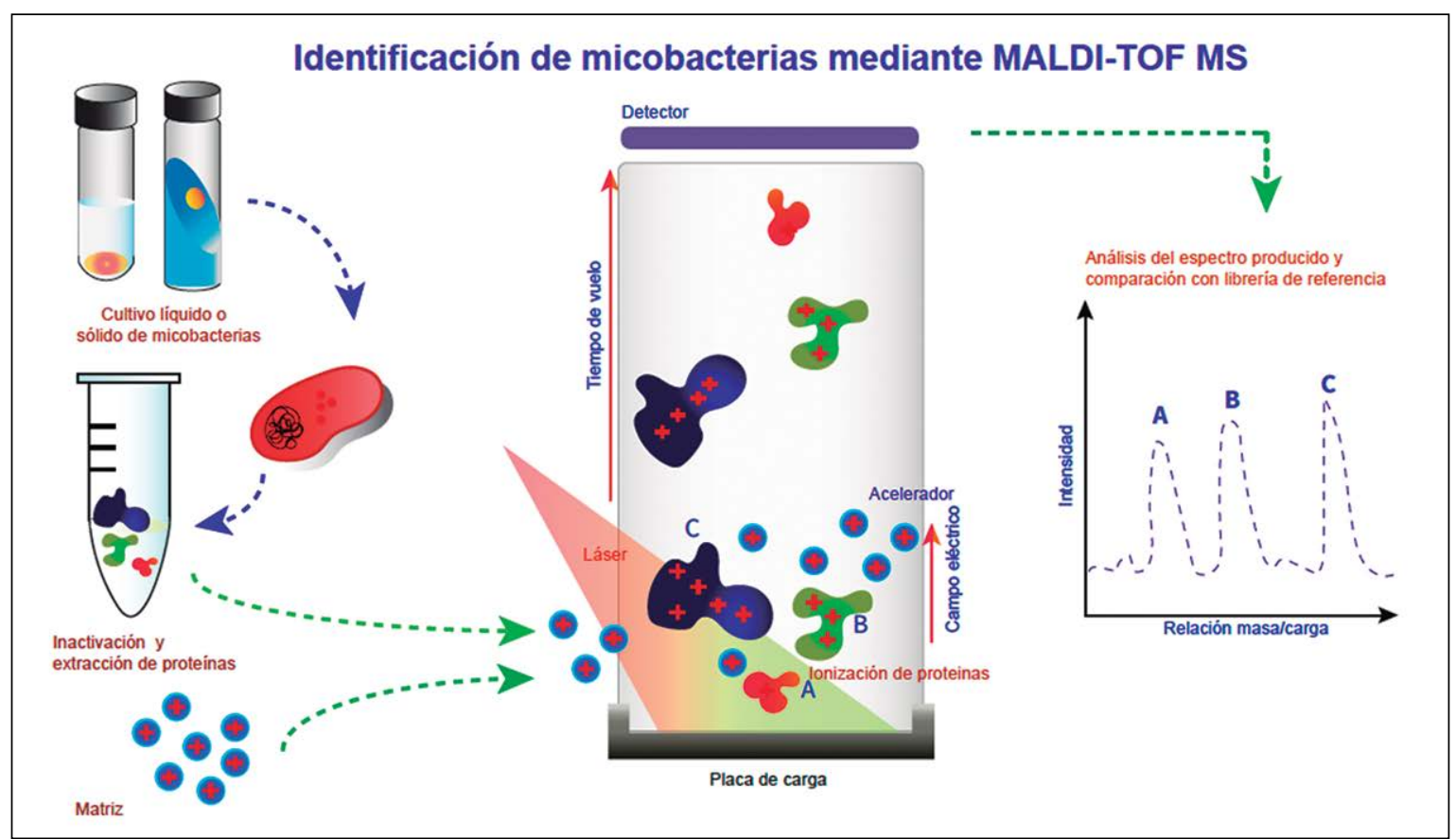

Tabla 1. Resumen de resultados

\begin{tabular}{lllll}
\hline Clasificación & Especie o complejo & \multicolumn{1}{c}{ Identificación } \\
MNTB & Crecimiento rápido & Complejo M. abscessus/chelonae & $4 / 4(100 \%)$ & $9 / 9(100 \%)$ \\
& & Complejo M. fortuitum & $2 / 2(100 \%)$ & \\
& M. mucogenicum & $2 / 2(100 \%)$ & \\
& M. neoaurum & $1 / 1(100 \%)$ & \\
\multirow{2}{*}{ Crecimiento lento } & Complejo M. avium/intracellulare & $5 / 11(45 \%)$ & $9 / 15(60 \%)$ \\
& & M. gordonae & $2 / 2(100 \%)$ & \\
& M. kanssasii & $1 / 1(100 \%)$ & \\
& Complejo M. terrae & $1 / 1(100 \%)$ & \\
MTB & Complejo M. tuberculosis & & $4 / 4(100 \%)$ \\
\hline
\end{tabular}

Identificación global 22/28 78,5\%.

MNTB: Mycobacterium no tuberculosis. MTB: Mycobacterium tuberculosis.
Germany). El espectro resultante fue analizado con el programa Biotyper 3 versión 3.1, utilizando la biblioteca para micobacterias (Mycobacteria library 4.0). Para una identificación a nivel de género y especie se consideró un score de correlación $\geq 1,8^{13,14}$.

\section{Resultados}

Utilizando el método de MALDI-TOF, se identificó correctamente a nivel de especie $78,5 \%$ de las aislados $(22 / 28)$ (Tabla 1). La identificación fue concordante en $100 \%(9 / 9)$ de las especies de MNTB de crecimiento rápido (complejo M. abscessus/chelonae (4/4), M. fortuitum (2/2), M. mucogenicum (2/2) у M. neoaurum (1/1)). En las especies de MNTB de crecimiento lento se obtuvo una identificación correcta en $60 \%$ de los aislados $(9 / 15)$ (complejo M. avium/intracellulare (5/11), M. gordonae (2/2), M. kansasii (1/1) y complejo M. terrae (1/1)). En el caso de MTBc, se identificó correctamente a $100 \%$ de los aislados (4/4). Las seis especies que no pudieron ser identificadas correspondieron al complejo M. avium/ intracellulare. En los seis casos el score fue $<1,4$, por lo que no correspondieron a identificaciones erróneas.

De las identificaciones a partir de cultivo sólido (n: 5), en $100 \%(5 / 5)$ se obtuvo un score $\geq 1,8$ y todas correspondieron a micobacterias de crecimiento rápido. En el caso de los cultivos líquidos se pudo identificar 74\% (17/23) de las aislados (Tabla 2). 


\begin{tabular}{|c|c|c|c|c|c|}
\hline & Identificación molecular & Identificación MALDI-TOF & Score & Nivel de identificación & Tipo de cultivo \\
\hline 1 & Complejo M. chelonae/absessus & M. chelonae & 2.011 & Especie & Sólido \\
\hline 2 & Complejo M. fortuitum & M. fortuitum & 2.213 & Especie & Líquido \\
\hline 3 & Complejo M. chelonae/absessus & M. abscessus & 1.947 & Especie & Sólido \\
\hline 4 & Complejo M. tuberculosis & Complejo M. tuberculosis & 2.126 & Especie & Líquido \\
\hline 5 & M. gordonae & M. gordonae & 2.153 & Especie & Líquido \\
\hline 6 & M. kansassii & M. kansassii & 1.963 & Especie & Líquido \\
\hline 7 & M. neoaurum & M. neoaurum & 1.885 & Especie & Sólido \\
\hline 8 & Complejo M. tuberculosis & Complejo M. tuberculosis & 2.289 & Especie & Líquido \\
\hline 9 & Complejo M. tuberculosis & Complejo M. tuberculosis & 2.006 & Especie & Líquido \\
\hline 10 & M. chelonae & M. chelonae & 2.126 & Especie & Sólido \\
\hline 11 & Complejo M. terrae & Complejo M. terrae & 2.282 & Especie & Líquido \\
\hline 12 & M. intracellulare & Grupo M. chimaera/intracellulare & 1.852 & Especie & Líquido \\
\hline 13 & Complejo M. fortuitum & M. fortuitum & 2.265 & Especie & Líquido \\
\hline 14 & Complejo M. tuberculosis & Complejo M. tuberculosis & 2.189 & Especie & Líquido \\
\hline 15 & M. avium & No identifica & - & - & Líquido \\
\hline 16 & M. gordonae & M. gordonae & 1.996 & Especie & Líquido \\
\hline 17 & M. avium & M. avium & 1.956 & Especie & Líquido \\
\hline 18 & M. intracellulare & Grupo M. chimaera/intracellulare & 2.036 & Especie & Líquido \\
\hline 19 & M. intracellulare & Grupo M. chimaera/intracellulare & 1.841 & Especie & Líquido \\
\hline 20 & M. avium & No identifica & - & - & Líquido \\
\hline 21 & M. abscessus & M. abscessus & 2.144 & Especie & Líquido \\
\hline 22 & M. mucogenicum & M. mucogenicum & 2.121 & Especie & Sólido \\
\hline 23 & M. avium & M. avium & 1.861 & Especie & Líquido \\
\hline 24 & M. intracellulare & No identifica & - & - & Líquido \\
\hline 25 & M. mucogenicum & M. mucogenicum & 2.015 & Especie & Líquido \\
\hline 26 & M. avium & No identifica & - & - & Líquido \\
\hline 27 & M. intracellulare & No identifica & - & - & Líquido \\
\hline 28 & M. intracellulare & No identifica & - & - & Líquido \\
\hline
\end{tabular}

\section{Discusión}

La diferenciación de especies micobacterianas estrechamente relacionadas es un desafío común. El uso de MALDI-TOF MS en laboratorios de microbiología clínica ha representado un gran avance en la identificación de bacterias y levaduras debido a que es un método rápido (aproximadamente 1 a $2 \mathrm{~h}$ en comparación con semanas de identificación fenotípica), exacto y simple ${ }^{15,16}$, que puede ser implementado en laboratorios de microbiología general; además, el costo de la identificación de MALDI-TOF MS es significativamente menor en comparación con otros métodos, incluidos la secuenciación genómica o técnicas bioquímicas y genera menos desperdicios asimilables o de riesgo biológico que otros métodos que utilizan muchos materiales desechables ${ }^{17}$.

En nuestro estudio, MALDI-TOF MS permitió una identificación correcta en $100 \%$ de las especies de micobacterias estudiadas, con excepción de M. avium/ intracellulare donde la tasa de éxito fue de $45 \%$ a nivel de especie, sin resultados discordantes. El menor rendimiento de la metodología para la identificación de M. avium/intracellulare se podría explicar debido a que existen puntos críticos que afectan la calidad del espectro obtenido y, en consecuencia la identificación, como es la inactivación previa de los microorganismos con alta probabilidad de desnaturalización proteica, la baja biomasa obtenida para el análisis y la probable interferencia por proteínas proce- 
dentes de los medios de cultivo en muestras obtenidas de medios líquidos, así como la falta de actualización de la base datos de comparación. Estas razones, en conjunto, son aspectos que han sido considerados en el momento de establecer el puntaje de corte para asignar género y especie a las muestras analizadas que, a diferencia de la bacteriología general donde se recomienda un corte de 2 para una identificación nivel de especie, en el caso de micobacterias sea de $1,8^{18}$. En cuanto a limitaciones de este estudio, destaca que no hay evaluación de todas las especies de micobacterias y que las determinaciones no fueron realizadas en duplicado, lo que debe considerarse en el futuro; además no se repitieron las determinaciones con score $<1,8$ dado que no se disponía de un segundo tubo de cultivo líquido positivo. Sin embargo, es necesario considerar que existen especies de MNTB estrechamente relacionados que históricamente se sabe poseen un espectro proteico cercano al límite de resolución de MALDI-
TOF MS como M. abscessus, M. mucogenicum y $M$. phocaicum, así como M. chimaera y M. intracellulare ${ }^{19}$, con lo que se puede explicar parcialmente la baja tasa de éxito de identificación de $M$. avium/intracellulare.

A pesar de que el costo inicial del equipo (espectrómetro de masas) es elevado, el gasto calculado en insumos por determinación (sin incluir costo de mano de obra y uso de otros equipos) es de aproximadamente US\$3, mientras que las técnicas moleculares pueden valer siete veces o más.

Es importante destacar que a pesar de no lograr el $100 \%$ de la identificación de los aislados analizados, MALDI-TOF MS no entregó resultados erróneos, por lo que se puede considerar como un método confiable de identificación. La simplicidad, rapidez y el bajo costo del método lo hacen recomendable para la identificación de micobacterias en un laboratorio de rutina que cuente con el equipo.

\section{Referencias bibliográficas}

1.- Matteelli A, Sulis G, Capone S, D'Ambrosio L, Migliori G B, Getahun H. Tuberculosis elimination and the challenge of latent tuberculosis. Presse Med 2017; 46, e13-e21. doi: 10.1016/j.lpm.2017.01.015.

2.- Nahid P, Menzies D. Update in tuberculosis and nontuberculous mycobacterial disease 2011. Am J Respir Crit Care Med 2012; 185: 1266-70. doi: $10.1164 / \mathrm{rccm} .201203-$ 0494UP.

3.- McBryde E S, Meehan M T, Doan T N, Ragonnet R, Marais B J, Guernier V, et al. The risk of global epidemic replacement with drug-resistant Mycobacterium tuberculosis strains. Int J Infect Dis 2017; 56: 14-20. doi: 10.1016/j.ijid.2017.01.031

4.- Griffith D E, Aksamit T R. Understanding nontuberculous mycobacterial lung disease: it's been a long time coming. 2016; F1000Research; 5: 2797. doi: 10.12688/ f1000research.9272.1.

5.- van Ingen J. Diagnosis of nontuberculous mycobacterial infections. Semin Respir Crit Care Med 2013; 34: 103-9. doi: 10.1055/s0033-1333569.

6.- $\quad$ Fan W.-T, Qin T-T, Bi R-R, Kang H-Q, Ma $\mathrm{P}, \mathrm{Gu}$ B. Performance of the matrix-assisted laser desorption ionization time-of-flight mass spectrometry system for rapid identification of streptococci: a review. Eur J Clin Microbiol Infect Dis 2017; 36: 1005-12. doi: 10.1007/ s10096-016-2879-2.

7.- Suarez S, Nassif X, Ferroni A. Applications of MALDI-TOF technology in clinical microbiology. Pathol. Biol. (Paris). 2015; 63: 43-52. doi: 10.1016/j.patbio.2014.10.002.

8.- Seng P, Rolain J-M, Fournier P E, La Scola B, Drancourt M, Raoult D. MALDI-TOFmass spectrometry applications in clinical microbiology. Future Microbiol 2010; 5: 173354. doi: $10.2217 / \mathrm{fmb} .10 .127$.

9.- Niitsuma K, Saito M, Koshiba S, Kaneko M. Identification of mycobacteria by matrixassisted laser desorption-ionization timeof-flight mass spectrometry-using reference strains and clinical isolates of Mycobacterium. Kekkaku 2014; 89: 555-63. doi: 10.1128/ JCM.01959-05.

10.- Şamlı A, İlki A. Comparison of MALDITOF MS, nucleic acid hybridization and the MPT64 immunochromatographic test for the identification of $M$. tuberculosis and nontuberculosis Mycobacterium species. New Microbiol 2016; 39: 259-63.

11.- Wattal C, Oberoi J K, Goel N, Raveendran R, Khanna S. Matrix-assisted laser desorption ionization time of flight mass spectrometry (MALDI-TOF MS) for rapid identification of micro-organisms in the routine clinical microbiology laboratory. Eur. J. Clin. Microbiol. Infect. Dis. 2017; 36, 807-12. doi: 10.1007/s10096-016-2864-9.

12.- Chedore P, Th'ng C, Nolan DH, Churchwell $\mathrm{GM}$, Sieffert DE, et al. Method for inactivating and fixing unstained smear preparations of Mycobacterium tuberculosis for improved laboratory safety. J Clin Microbiol 2002; 40: 4077-80. doi: 10.1128/JCM.40.11.40774080.2002 .

13.- Lotz A, Ferroni A, Beretti J-L, Dauphin B,
Carbonnelle E, Guet-Revillet H, et al. Rapid identification of mycobacterial whole cells in solid and liquid culture media by matrixassisted laser desorption ionization-time of flight mass spectrometry. J Clin Microbiol 2010; 48: 4481-6. doi: 10.1128/JCM.01397-10.

14.- Inactivation I O. Standard Operating Procedure Mycobacteria Extraction (MycoEX) Method MALDI Biotyper. 3, 3-6 (2014).

15.- Patel R. MALDI-TOF MS for the diagnosis of infectious diseases. Clin Chem 2015; 61, 100. doi: 10.1373/clinchem.2014.221770.

16.- Drake R, Boggs S R, Drake S K. Pathogen identification using mass spectrometry in the clinical microbiology laboratory. J. Mass Spectrom. 2011; 46, 1223. doi: 10.1002/ jms.2008.

17.- Balada-Llasat M, Kamnoj K, Pancholi P. Identification of mycobacteria from solid and liquid media by matrix-assisted laser desorption ionization-time of flight mass spectrometry in the clinical laboratory. J Clin Microbiol 2013; 51: 2875. doi: 10.1002/jms.2008.

18.- van Eck K, Faro D, Wattenberg M, de Jong A, Kuipers S, van Ingen J, et al. Matrix-assisted laser desorption ionization-time of flight mass spectrometry fails to identify nontuberculous mycobacteria from primary cultures of respiratory samples. J Clin Microbiol 2016; 54, 1915. doi: 10.1128/JCM.00304-16.

19.- Saleeb P G, Drake S K, Murray P R, Zelazny A M. Identification of mycobacteria in solid-culture media by matrix-assisted laser desorption ionization-time of flight mass spectrometry. J Clin Microbiol 2017; 49: 790 1790-4 doi: 10.1128/JCM.02135-10 